\title{
Analysis of risk factors and clinical indicators in bloodstream infections among patients with hematological malignancy : a retrospective study
}

\author{
Yating Ma \\ Chinese PLA General Hospital \\ Ming Yang \\ Central South University Third Xiangya Hospital \\ Jinfeng Bao \\ Chinese PLA General Hospital \\ Chengbin Wang ( $\sigma$ wangcbin301@163.com ) \\ PLA General Hospital
}

\section{Research article}

Keywords: Bacteremia, Hematological malignancy, Risk factors, Interleukin-6, D-dimer

Posted Date: October 2nd, 2020

DOI: https://doi.org/10.21203/rs.3.rs-85374/v1

License: (c) (i) This work is licensed under a Creative Commons Attribution 4.0 International License. Read Full License 


\section{Abstract}

Background: The incidence of bloodstream infection caused by bacteremia is more common in patients with hematological malignancy. It is important to distinguish infectious episodes from non-infectious episodes. The present study was aimed to describe epidemiology and clinical indexes for in-hospital infection of hematological malignancy patients.

Methods: Single-center retrospective research was performed on hematological malignancy patients admitted to our hospital from July 2015 to March 2018. Laboratory and clinical information from 322 febrile patients were acquired. These episodes were divided by blood culture results into two groups: (1) blood culture positive group, (2) blood culture negative group.

Results: In the 322 febrile cases, 81 (25.2\%) patients were blood culture positive, and among them, Gram-negative bacteria (51.9\%) were more isolated than Gram-positive bacteria (32.1\%) and fungi (7.4\%). Gram-negative bacteria were more likely to have a drug resistance than Grampositive bacteria. Independent risk factors revealed that patients with complications, high levels of procalcitonin (PCT), glucose, interleukin-6 (IL-6) and d-dimer (D-D), and low concentration of albumin were correlated with occurrence of infection. PCT, IL-6 and D-D performed well in differentiating not only the infection group from the non-infection group, but also in the Gram-negative group from the Gram-positive group with the areas under the curve all above 0.75 .

Conclusions: We analyzed the risk factors for bloodstream infection in patients with hematological malignancy, the distribution of bacteria, antibiotics resistance and the changes of clinical parameters. This single-center retrospective study may provide clinicians insight to the diagnosis and treatment of infection.

\section{Background}

Blood stream infection (BSI) remains a common concern for cancer patients, especially hematological malignancy patients who often receive chemotherapy or hematopoietic stem cell transplanting [1, 2]. Distinguishing infectious from non-infectious episodes is more complicated in hematological malignancy patients. Fever is a common symptom of infected patients and its early recognition is necessary to offer timely antibiotic therapy [3]. As a result, sufficient and immediate clinical laboratory results are critical for patients with suspected bacterial infection.

Blood culture, the gold standard of infection diagnosis, often takes 3-5 days and is limited by the relatively low positive rate, which restricts the use in early diagnosis [4]. Patients with infection are often accompanied with changes of biochemical indexes, such as albumin (ALB), glucose (GLU), alanine transaminase (ALT), and aspartate transaminase (AST). Some clinical parameters, including white blood cell (WBC), platelets (PLT), C reaction protein (CRP), procalcitonin (PCT), interleukin-6 (IL-6) and d-dimer (D-D) are commonly used in diagnosing infection [5-9]. However, these parameters have not been widely studied in hematological malignancy patients. Therefore, in this study, the clinical characteristics, bacterial distribution, antibiotics use and resistance, inflammation biomarkers and risk factors of 322 hematologic malignancy patients with febrile episodes were systematically and retrospectively analyzed to predict bacterial infection.

\section{Methods}

\subsection{Enrollment of patients and clinical data collection}

This retrospective study was conducted at the PLA General Hospital with approval by the Ethical Committee (No.S2018-207002). All adult patients diagnosed as hematological malignancies accompanied with febrile syndrome and undergoing blood culture were recorded from July 2015 to March 2018. Totally 322 patients were included who had all clinical and laboratory data, such as WBC, PLT, CRP, PCT, IL-6, D-D, ALB, GLU, ALT, AST, blood culture results, antibiotic use and resistance. These patients suffered fevers with body temperature above $37.5^{\circ} \mathrm{C}$, and their bloods were collected for blood culture. Bacterial BSI was diagnosed with blood culture results, indicating the types of microorganisms causing the infection. .

\subsection{Laboratory tests}

Blood samples were collected when patients had fever, cough, chill and related infection syndrome to measure WBC, PLT, CRP, PCT, IL-6, D-D, ALB, GLU, ALT and AST levels. Blood (5-10 mL) was sampled 2 to 3 times and cultured in Bactec Plus Aerobic/F (BD) bottles. The species of blood culture isolates were recognized by MALDI-TOF-MS (Bio-Merieux, Marcy l'Etoile, France). Antimicrobial susceptibility was tested by a Vitek 2 system (Bio-Mérieux). WBC and PLT were measured with a Sysmex-XN9000 haematology analyzer. Serum CRP concentration was detected using a PuMen CRP analyzer. PCT was tested in Roche cobas 8000. ALB, GLU, ALT and AST were measured in Roche cobas c501. IL-6 was tested in Roche E411. D-D was determined in a STAGO coagulation analyzer (France).

\subsection{Statistical analysis}

Continuous data were expressed as median and range or 95\% confidence interval ( $\mathrm{Cl})$, and categorical data as number and percentages. Categorical data were compared by Chi-square test, and abnormally arranged variables by Mann-Whitney $U$ test. The independent indices 
forecasting bacteremia in hematologic patients with febrile episode were identified by logistic regression. Any index with $\mathrm{P}<0.2$ in univariate analysis was sent to logistic regression. Reliability of each index for bacteremia diagnosis was assessed using receiver-operating characteristic (ROC) curve and area under the curve (AUC). Diagnostic accuracy including sensitivity and specificity was computed using cut-off values. The optimal diagnostic cutoff level was found using Youden's index. All analyses were performed on SPSS 19.0 (IBM Corp., Armonk, NY, USA) and Graphad Prism 6.0 (GraphPad Software, La Jolla, CA, USA). P 0.05 implied significance.

\section{Results}

\subsection{Clinical characteristics of patients with hematological malignancies}

Totally 322 febrile episodes were included and separated by blood culture results into a positive (infection) group and a negative (non-infection) group. The general characteristics were shown in Table 1. There were more male patients in these two groups. Median ages were 44 and 43 years old respectively. The main primary diseases of these two groups were acute myeloid leukemia (AML), lymphoma, acute lymphoblastic leukemia (ALL), and myelodysplastic syndrome (MDS). Infected patients intended to have more complications (88.9\%) than non-infected patients $(68.9 \%)$. Moreover, there were high percentages of diabetes and smoking in the infection group. The length of hospital stay was not much different between the two groups.

Table 1

Comparison of clinical characteristics between blood culture $(+)$ and blood culture $(-)$ group

\begin{tabular}{|c|c|c|}
\hline Characteristics & Blood culture $(+)(n=81)$ & Blood culture $(-)(n=241)$ \\
\hline Sex (Male), no(\%) & $49(60.5)$ & $148(61.4)$ \\
\hline Age, median (range) & $44(21-87)$ & $43(18-91)$ \\
\hline \multicolumn{3}{|c|}{ Underlying disease, no\% } \\
\hline AML & $35(43.2)$ & $98(40.7)$ \\
\hline ALL & $9(11.1)$ & $43(17.8)$ \\
\hline Lymphoma & $23(28.4)$ & $63(26.1)$ \\
\hline MDS & $4(4.9)$ & $16(6.6)$ \\
\hline MM & $5(6.2)$ & $9(3.7)$ \\
\hline AA & $1(1.3)$ & $7(2.9)$ \\
\hline Others & $4(4.9)$ & $5(2.2)$ \\
\hline \multicolumn{3}{|l|}{ Complications, no\% } \\
\hline Yes & 72 (88.9) & $166(68.9)$ \\
\hline No & $9(11.1)$ & $75(31.1)$ \\
\hline \multicolumn{3}{|l|}{ Diabetes, no\% } \\
\hline Yes & $9(11.1)$ & $21(8.7)$ \\
\hline No & $72(88.9)$ & $220(91.3)$ \\
\hline \multicolumn{3}{|l|}{ Smoke, no\% } \\
\hline Yes & $25(30.9)$ & $54(22.4)$ \\
\hline No & $56(69.1)$ & 187 (77.6) \\
\hline \multicolumn{3}{|l|}{ Days in hospital, no\% } \\
\hline$\leq 30$ & $36(44.4)$ & 105 (43.6) \\
\hline$>30$ & $45(55.6)$ & $136(56.4)$ \\
\hline
\end{tabular}

\subsection{Distribution of pathogens and the use of antibiotics and resistance}


The distribution of pathogens was shown in Fig. 1. The most frequent Gram-negative bacterium in blood culture was Escherichia coli (21\%), followed by Klebsiella pneumoniae (8.6\%), Pseudomonas aeruginosa (6.2\%) and Brucella (6.2\%). The frequent Gram-positive bacteria were Staphylococci hominis (S.homins) (12.3\%), Staphylococcus epidermidis (S.epidermidis) (7.4\%), Coagulase-negative Staphylococci (CNS) (3.7\%), Staphylococci. capitis (S.capitis) (2.5\%) and Staphylococci hemolyticus (S.hemolyticus) (2.5\%). Fungemia occupied $7.4 \%$ in the infection group; multiple bacterial $(\geq 2)$ infection occurred in $8.6 \%$ episodes. Because of the definite blood culture results, the antibiotics use was more targeted. The four major antibiotics used in the infection group were meropenem (25\%), imipenem (23.4\%) cephalosporins (15.6\%) and vancomycin (14.1\%) (Table 2). In the non-infection group, however, the results were unclear which pathogens caused infection, and empirical medication of antibiotics was implemented, including cephalosporins (33.6\%), imipenem (24.2\%) and meropenem (16.4\%). Gramnegative bacteria were more likely to have a drug resistance than Gram-positive bacteria (Table 3). Both Gram-negative or Gram-positive bacteria were resistant against penicillins and quinolones, and especially E.coli was most resistant against quinolones. For cephalosporins resistance, it often occurred in Gram-negative bacteria. Resistance against tetracyclines, erythromycin and clindamycin occurred in most Grampositive bacteria than Gram-negative bacteria. Fungi were likely itraconazole-resistant (Table 3).

Table 2

The use of antibiotics in different groups

\begin{tabular}{|lll|}
\hline Antibiotics use & Blood culture $(+)(\mathbf{n}=\mathbf{8 1})$ & Blood culture $(-)(\mathbf{n = 2 4 1 )}$ \\
\hline Antibiotics use all & 64 & 128 \\
\hline Meropenem, no\% & $16(25)$ & $21(16.4)$ \\
\hline Imipenem, no\% & $15(23.4)$ & $31(24.2)$ \\
\hline Cephalosporins, no\% & $10(15.6)$ & $43(33.6)$ \\
\hline Moxifloxacin, no\% & $4(6.3)$ & $3(2.3)$ \\
\hline Vancomycin, no\% & $9(14.1)$ & $6(4.7)$ \\
\hline Levofloxacin, no\% & $3(4.7)$ & $3(2.3)$ \\
\hline Teicoplanin, no\% & $0(0)$ & $10(7.8)$ \\
\hline Antifungal drugs, no\% & $2(3.1)$ & $9(7.1)$ \\
\hline Others, no\% & $5(7.8)$ & $2(1.6)$ \\
\hline
\end{tabular}


Table 3

Resistance of antibiotics in different pathogens in infection group

\begin{tabular}{|c|c|c|c|c|c|c|c|c|c|}
\hline \multirow{2}{*}{$\begin{array}{l}\text { Antibiotics } \\
\text { resistance }\end{array}$} & \multicolumn{4}{|c|}{ Gram negative bacteria } & \multicolumn{4}{|c|}{ Gram positive bacteria } & \multirow{2}{*}{$\begin{array}{l}\text { Fungi } \\
\text { Candida } \\
\text { Albicans } \\
\text { (5) }\end{array}$} \\
\hline & $\begin{array}{l}\text { E. coli } \\
\text { (19) }\end{array}$ & $\begin{array}{l}\text { K. } \\
\text { Pneumoniae } \\
\text { (8) }\end{array}$ & $\begin{array}{l}P \\
\text { Aeruginosa } \\
\text { (6) }\end{array}$ & Others(13) & $\begin{array}{l}\text { S. } \\
\text { Hominis } \\
(11)\end{array}$ & $\begin{array}{l}\text { S. } \\
\text { Epidermidis } \\
(6)\end{array}$ & $\begin{array}{l}\text { Other } \\
\text { Staphylococcus } \\
\text { (7) }\end{array}$ & $\begin{array}{l}\text { Others } \\
\text { (5) }\end{array}$ & \\
\hline Penicillins, no\% & $\begin{array}{l}9 \\
(47.4)\end{array}$ & $3(37.5)$ & $3(50)$ & $2(15.4)$ & $8(72.7)$ & $3(50)$ & $2(28.6)$ & $1(20)$ & NA \\
\hline $\begin{array}{l}\text { Cephalosporins, } \\
\text { no\% }\end{array}$ & $\begin{array}{l}8 \\
(42.1)\end{array}$ & $2(25)$ & $4(66.7)$ & $4(30.8)$ & $1(9.1)$ & 0 & 0 & 0 & NA \\
\hline Quinolones, no\% & $\begin{array}{l}12 \\
(63.2)\end{array}$ & $1(12.5)$ & 0 & $1(7.7)$ & $6(54.5)$ & $3(50)$ & $2(28.6)$ & $2(40)$ & NA \\
\hline Sulbactam, no\% & $\begin{array}{l}2 \\
(10.5)\end{array}$ & 0 & 0 & 0 & 0 & 0 & 0 & 0 & NA \\
\hline Aztreonam, no\% & $\begin{array}{l}3 \\
(15.8)\end{array}$ & $1(12.5)$ & 0 & $2(15.4)$ & 0 & 0 & 0 & 0 & NA \\
\hline Gentamicin, no\% & $\begin{array}{l}8 \\
(42.1)\end{array}$ & 0 & 0 & $1(7.7)$ & 0 & 0 & 0 & 0 & NA \\
\hline $\begin{array}{l}\text { Sulfonamides, } \\
\text { no\% }\end{array}$ & $\begin{array}{l}3 \\
(15.8)\end{array}$ & $1(12.5)$ & $1(16.7)$ & 0 & $1(9.1)$ & 1 & 0 & $1(20)$ & NA \\
\hline Furadantin, no\% & 0 & 0 & $2(33.3)$ & $1(7.7)$ & 0 & 0 & 0 & 0 & NA \\
\hline $\begin{array}{l}\text { Tetracyclines, } \\
\text { no\% }\end{array}$ & 0 & 0 & 0 & 0 & $5(45.5)$ & $1(16.7)$ & 0 & $1(20)$ & NA \\
\hline $\begin{array}{l}\text { Erythromycin, } \\
\text { no\% }\end{array}$ & 0 & 0 & 0 & 0 & 7 & $4(66.7)$ & $1(14.3)$ & 0 & NA \\
\hline Clindamycin,no\% & 0 & 0 & 0 & 0 & 0 & $1(16.7)$ & $1(14.3)$ & 0 & NA \\
\hline $\begin{array}{l}\text { Itraconazole, } \\
\text { no\% }\end{array}$ & NA & NA & NA & NA & NA & NA & NA & NA & $1(20)$ \\
\hline
\end{tabular}

\subsection{Risk factors for BSI in hematological malignancies patients}

In univariate analysis (Table 4), some significant variables $(\mathrm{P}<0.2)$ were involved and adjusted in the multivariate analysis. Age, diabetes, inpatient days, ALT and AST were insignificant in infection, which may be due to the number of patients or the statistic analysis. Multivariate analysis confirmed six variables were significantly associated with infection: having complications (OR [95\% CI]: 3.459 [1.183-10.109]; $P=$ 0.023), PCT (1.29 [1.107-1.503]; P = 0.001), ALB (0.885 [0.795-0.92]; P < 0.001), GLU (1.297 [1.092-1.539]; P = 0.003), IL-6 (1.048 [1.025-1.07]; $\mathrm{P}<0.001)$ and D-D (1.048 [1.025-1.07]; $\mathrm{P}<0.001)$ (Table 5). No such association was observed in sex, smoke, CRP or PLT in multivariate analysis. 
Table 4

Univariate analysis of the risk factors for infection and non-infection among 322 episodes in patients with hematologic malignancies

\begin{tabular}{|c|c|c|c|c|c|}
\hline \multirow[t]{2}{*}{ Variables } & \multicolumn{2}{|c|}{ Blood culture $(+)(n=81)$} & \multicolumn{2}{|c|}{ Blood culture $(-)(n=241)$} & \multirow[t]{2}{*}{ P-value } \\
\hline & $\mathrm{N}$ & $\%$ & $\mathrm{~N}$ & $\%$ & \\
\hline Age, year & & & & & 0.884 \\
\hline$<60$ & 67 & 82.7 & 213 & 88.4 & \\
\hline$\geq 60$ & 14 & 17.3 & 28 & 11.6 & \\
\hline Sex & & & & & 0.193 \\
\hline Male & 49 & 60.5 & 148 & 61.4 & \\
\hline Female & 32 & 39.5 & 93 & 38.6 & \\
\hline Diabetes & & & & & 0.522 \\
\hline Yes & 9 & 11.1 & 21 & 8.7 & \\
\hline No & 72 & 88.9 & 220 & 91.3 & \\
\hline Smoke & & & & & 0.128 \\
\hline Yes & 25 & 30.9 & 54 & 22.4 & \\
\hline No & 56 & 69.1 & 187 & 77.6 & \\
\hline Complications & & & & & 0.001 \\
\hline Yes & 72 & 88.9 & 166 & 68.9 & \\
\hline No & 9 & 11.1 & 75 & 31.1 & \\
\hline Days in hospital & & & & & 0.891 \\
\hline$\leq 30$ & 36 & 44.4 & 105 & 43.6 & \\
\hline$>30$ & 45 & 55.6 & 136 & 56.4 & \\
\hline Variables & Bloc & $=81)$ (median \pm quantile) & $\begin{array}{l}\text { Bloo } \\
\text { (med }\end{array}$ & $\begin{array}{l}\text { e }(-)(n=241) \\
\text { Jantile })\end{array}$ & P-value \\
\hline WBC & 1.24 & & 2.71 & 52.23) & 0.036 \\
\hline PLT & 31( & & $39(2$ & & 0.016 \\
\hline CRP & $8(4$ & & 4.35 & 3.93) & $<0.001$ \\
\hline PCT & 2.65 & & 0.23 & $0.67)$ & $<0.001$ \\
\hline ALB & 31.5 & & 35.3 & 39.2) & $<0.001$ \\
\hline GLU & 6.95 & & 5.3( & 64) & $<0.001$ \\
\hline ALT & 19.5 & & 18.7 & $-31.25)$ & 0.475 \\
\hline AST & 16.2 & & 17.1 & $-30.2)$ & 0.679 \\
\hline IL-6 & 32.1 & & 7.58 & 22) & $<0.001$ \\
\hline D-D & 3.1 & & 0.61 & 1.1) & $<0.001$ \\
\hline
\end{tabular}


Table 5

Multivariate analysis of the risk factors for infection/non-infection among 322 episodes in patients with hematologic malignancies

\begin{tabular}{|llll|}
\hline Variables & \multicolumn{3}{l|}{ Infection/non-infection } \\
\hline & OR & $95 \% \mathrm{Cl}$ & P-value \\
\hline Complications & 3.459 & $1.183-10.109$ & 0.023 \\
PCT & 1.29 & $1.107-1.503$ & 0.001 \\
ALB & 0.885 & $0.795-0.92$ & $<0.001$ \\
\hline GLU & 1.297 & $1.092-1.539$ & 0.003 \\
\hline IL-6 & 1.048 & $1.025-1.07$ & $<0.001$ \\
\hline D-D & 1.19 & $1.06-1.335$ & 0.003 \\
\hline
\end{tabular}

\subsection{Diagnostic accuracy of indicators for bacteremia detection}

ROC curves of WBC, PLT, CRP, PCT, IL-6, D-D, ALB, GLU, ALT and AST levels were plotted to diagnose bacteremia in the 322 febrile episodes (Figure. 2). Among these indicators, the AUCs of PCT, IL- 6 and D-D were $0.8473,0.7937$ and 0.8613 respectively, indicating higher diagnostic accuracy. Table 6 showed the diagnostic sensitivity and specificity of these indexes with the best cut-off value. When we analyzed WBC, CRP, PCT, IL-6 and D-D in differentiating Gram-negative and Gram-positive infection, PCT, IL-6 and D-D all demonstrated high diagnostic accuracy, with AUC of $0.7834,0.8182$ and 0.8951 , respectively. However, neither WBC nor CRP can effectively differentiate these two groups (Fig. 3).

Table 6

Sensitivity and specificity of parameters in differentiating bacteremia group from non-bacteremia group

\begin{tabular}{|llll|}
\hline Variables & Cut-off & Sensitivity (\%) & Specificity (\%) \\
\hline WBC & 0.895 & 44.44 & 73.44 \\
\hline PLT & 58.5 & 80.25 & 39 \\
CRP & 5.30 & 67.9 & 60.58 \\
\hline PCT & 0.99 & 77.78 & 81.33 \\
\hline ALB & 31.75 & 51.85 & 79.67 \\
\hline GLU & 6.780 & 53.09 & 77.59 \\
\hline ALT & 44.50 & 25.93 & 85.06 \\
\hline AST & 20.95 & 41.98 & 63.49 \\
\hline IL-6 & 18.15 & 66.67 & 80.08 \\
\hline D-D & 0.8850 & 88.89 & 70.95 \\
\hline
\end{tabular}

\section{Discussion}

Hematological patients are more vulnerable to pathogen infection because of long-term hospitalization, chemotherapy-induced immune suppression and antibiotic exposure, which lead to higher mortality. The common symptom of infection is fever, though noninfectious febrile episodes are often seen [2]. The gold standard of infection test is blood culture, but its application in early diagnosis is limited by huge timeconsumption and low positive rate. Therefore, it is important to distinguish infectious episodes from noninfectious patients, which provides appropriate and immediate antibiotic therapy. This retrospective study was aimed to analyze the differences in clinical characteristics, pathogens distribution, antibiotics use and resistance, clinical common indicators and diagnostic efficiency between bacterial positive patients and bacterial negative patients.

We enrolled 322 patients with hematological malignancy, including 81 bacteremia (+) and 241 bacteremia (-) patients. The positive rate of $25.2 \%$ was higher than other reports from different studies, which may be due to our objects were hematological patients who were more likely to have infection because of their lower immunity $[10,11]$. AML, lymphoma and ALL were the most common basic diseases in the two groups, 
which are consistent with other studies [1, 12-14]. In the blood culture positive group, more Gram-negative bacteria (51.9\%) were isolated than Gram-positive bacteria (32.1\%) and fungi (7.4\%), indicating that clinicians should attach great importance to infection by Gram-negative bacteria. Meanwhile, G- bacteria were more likely resistant against penicillins, cephalosporins and quinolones, due to the abuse of broadspectrum antibiotics, thus bringing great challenge to treatment $[14,15]$. As reported, $\mathrm{G}+$ bacteria were more frequent in some hospitals, even more than G- bacteria, with a high prevalence in clinical laboratory test [6]. Antibiotics resistance in $\mathrm{G}+$ bacteria was more common in penicillins, quinolones, tetracyclines and erythromycin in the present study. Our results were similar with other reports that empirical antibiotics treatment would increase resistance rate, bringing huge burden to clinical staff and patients $[3,6]$. According to blood culture and antibiotics resistance results, meropenem, imipenem and cephalosporins were used more frequently because they were all advanced antibiotics to treat infection. Patients without specific infection often receive empirical antibiotics treatment, including these advanced antibiotics, which often cause resistance.

Multivariate logistic analysis found that patients with complications, high PCT, GLU, IL-6 and D-D, and low ALB were more likely to have infection. As we all know, hematological malignancy patients are often accompanied with complications due to their immunocompromised status [1]. Moreover, patients with low ALB concentration are more likely to have lower immunity to resist bacterial infection [16, 17]. The indicators, including PCT, IL-6 and D-D, are inflammation biomarkers to assist in infection diagnosis. In a word, changes of the above factors might indicate the occurrence of infection.

Inflammation biomarkers often serve as assistant indicators in diagnosis, including WBC, PLT, CRP, PCT, IL-6 and D-D. We used ROC curves to investigate the diagnostic efficiency of these biomarkers. WBC is commonly used to indicate inflammation with the level increasing, but we found WBC counts were lower in the bacteremia group. The reason may be that the hematological patients under lower immunity status were accompanied with chemotherapy or marrow suppression. Meanwhile, the sensitivity and specificity of WBC were relatively low, which were insufficient for diagnosis of infection. Platelets, circulating blood cells from megakaryocytes, are pivotal in many physical activities, including clot formation, stop of bleeding, innate immune reply, and inflammation. As reported, PLT plays an important role not only in hemostasis, but also in antibacterial [18]. Platelets have a direct antibacterial defense effect through recognizing microbial antigens and secreting antimicrobial peptides and kinocidins, which can enhance innate immune effectors (including complement, neutrophils) and coordinate adaptive immunity (APS, T cells and B cells) [19]. However, in our study, PLT levels decreased in the bacteremia group compared with the non-bacteremia group with an AUC of 0.5803 to differentiate these two groups and the specificity was too low, which needs further research. Moreover, CRP, PCT, IL-6 and D-D levels significantly rose in the bacteremia group than the non-bacteremia group and performed well in differentiating these two groups. In comparison, PCT, IL-6 and D-D outperformed CRP in differentiating G-/+ groups, with AUC all above 0.75. CRP and PCT are commonly applied for infection detection. However, CRP concentrations often increase in non-infectious complications and are nonspecific for infection [20]. PCT is rapidly produced by thyroid gland C cells and several other types of cells, and is stimulated by bacterial endotoxins, lipopolysaccharids or inflammatory modulators (e.g. tumor necrosis factor-a, IL-6, IL-1) [21]. PCT reportedly can distinguish infection from noninfection. Yang et al. also found that PCT outperformed CRP in separating G- and G + infection in hematological malignancy patients [1, 22]. IL6 secreted into the blood initiates division of both B cells and T cells and generation of antibodies and acute stage proteins during acute stage infections. IL-6 is also involved in the activation of cytokine cascade in sepsis. IL-6 seems helpful in early assessment of sepsis and is related to disease severity $[8,9]$. Our results demonstrated that IL-6 performed well in differentiating not only infections from non-infections, but also Gfrom $\mathrm{G}+$ patients, which were consistent with previous studies [21, 23]. D-D, which results from fibrin degradation by fibrinolysis, is commonly used to screen venous thromboembolism clinically. In recent years, the relationship between inflammatory cytokines and biomarkers of the coagulation system has been widely studied, especially in critical patients with sepsis [7, 24-26]. Previous study found that the D-D level increased in pediatric hematological patients with E.coli bacteremia, which may be a clue for physicians to distinguish patients at higher risk for shock and mortality [7]. Though our data were all from hematological patients, D-D level increased in the bacteremia patients with a sensitivity of $88.89 \%$ and specificity of $70.95 \%$, and performed well in distinguishing G- or G + patients. However, some reports demonstrate no significant diagnostic value for patients in early phases of sepsis [27]. Thus, the function of D-D needs further research.

This retrospective study has several limitations. First, it was conducted at a single center hospital, which may not be generalizable to other settings. Second, we can not compare the laboratory parameters with clinical course due to some insufficient data from patients. Despite these limitations, our study may serve as a reference to guide clinicians in treating patients with bacterial infection, especially hematological episodes.ith prognosis of different conditions.

\section{Conclusion}

In summary, G- bacteria are more prevalent in febrile patients with hematological malignancy and often develop antibiotics resistance. Empirical antibiotic use may help anti-infection, but also form resistance. Meropenem, imipenem and cephalosporins show higher activity for the infection. Moreover, our findings demonstrated the risk factors for bacteremia in hematological diseases, including complications, low ALB concentration, high GLU, PCT, IL-6 and D-D levels, which may indicate the occurrence of infection. PCT, IL-6 and D-D are valuable indicators to aid infection diagnosis. Further studies are needed to validate these parameters in patients with prognosis of different conditions.

Page $8 / 12$ 


\section{Abbreviations}

BSI: Bloodstream infection; ALB: albumin; GLU: glucose; ALT: alanine transaminase; AST: aspartate transaminase; WBC: white blood cell; PLT: platelets; CRP: C reaction protein; PCT: procalcitonin; IL-6: interleukin-6; D-D: d-dimer

\section{Declarations}

\section{Ethics approval and consent to participate}

This study was conducted at the PLA General Hospital with approval by the Ethical Committee (No.S2018-207002).

\section{Consent for publication}

Not applicable.

\section{Availability of data and materials}

The datasets used and/or analyzed during the study are available from the corresponding author on reasonable request.

\section{Competing interests}

There are no conflicts of interest to declare.

\section{Funding}

This study was supported by the National Natural Science Foundation of China [grand number 81871731].

\section{Authors' contributions}

Yating Ma did collection and analysis of data and prepared the manuscript. Ming Yang collected clinical data. Jinfeng Bao did final check about data analysis and original work. Chengbin Wang did methodology designing and reviewing final check about manuscript. All authors have provided final approval for the final version of the manuscript.

\section{Acknowledgments}

The authors would like to thank PLA General Hospital for giving us the repository of clinical data about our study.

\section{References}

1. Yang M, Choi SJ, Lee J, Lee DG, Kim Y-J, Park Y-J, Oh E-J: Serum procalcitonin as an independent diagnostic markers of bacteremia in febrile patients with hematologic malignancies. PLoS One 2019, 14(12):e0225765.

2. Russo R, Mendes ET, Levin AS, Dulley F, Oliveira MS, Shikanai-Yasuda MA, Costa SF: Bloodstream infection in hematopoietic stem cell transplantation outpatients: risk factors for hospitalization and death. Rev Inst Med Trop Sao Paulo 2018, 61:e3.

3. Kagan E, Salgado CD, Banks AL, Marculescu CE, Cantey JR: Peripherally inserted central catheter-associated bloodstream infection: Risk factors and the role of antibiotic-impregnated catheters for prevention. Am J Infect Control 2019, 47(2):191-195.

4. Ma Y, Wen X, Kong Y, Chen C, Yang M, He S, Wang J, Wang C: Identification of New Peptide Biomarkers for Bacterial Bloodstream Infection. Proteomics Clin Appl 2020, 14(2):e1900075.

5. Shao IY, Elkind MSV, Boehme AK: Risk Factors for Stroke in Patients With Sepsis and Bloodstream Infections. Stroke 2019, 50(5):10461051.

6. El Haddad H, Chaftari A-M, Hachem R, Chaftari P, Raad II: Biomarkers of Sepsis and Bloodstream Infections: The Role of Procalcitonin and Proadrenomedullin With Emphasis in Patients With Cancer. Clin Infect Dis 2018, 67(6):971-977.

7. Schwameis M, Steiner MM, Schoergenhofer C, Lagler H, Buchtele N, Jilma-Stohlawetz P, Boehm T, Jilma B: D-dimer and histamine in early stage bacteremia: A prospective controlled cohort study. Eur J Intern Med 2015, 26(10):782-786.

8. Fioretto JR, Martin JG, Kurokawa CS, Carpi MF, Bonatto RC, Ricchetti SMQ, de Moraes MA, Padovani CR: Interleukin-6 and procalcitonin in children with sepsis and septic shock. Cytokine 2008, 43(2):160-164.

9. Hack CE, De Groot ER, Felt-Bersma RJ, Nuijens JH, Strack Van Schijndel RJ, Eerenberg-Belmer AJ, Thijs LG, Aarden LA: Increased plasma levels of interleukin-6 in sepsis. Blood 1989, 74(5):1704-1710.

10. Shao S-L, Cong H-Y, Wang M-Y, Liu P: The diagnostic roles of neutrophil in bloodstream infections. Immunobiology 2020, 225(1):151858. 
11. Zhu S, Kang Y, Wang W, Cai L, Sun X, Zong Z: The clinical impacts and risk factors for non-central line-associated bloodstream infection in 5046 intensive care unit patients: an observational study based on electronic medical records. Critical care (London, England) 2019 , 23(1):52.

12. Zhang Y, Guo L-Y, Song W-Q, Wang Y, Dong F, Liu G: Risk factors for carbapenem-resistant K. pneumoniae bloodstream infection and predictors of mortality in Chinese paediatric patients. BMC Infect Dis 2018, 18(1):248.

13. Lien M-Y, Chou C-H, Lin C-C, Bai L-Y, Chiu C-F, Yeh S-P, Ho M-W: Epidemiology and risk factors for invasive fungal infections during induction chemotherapy for newly diagnosed acute myeloid leukemia: A retrospective cohort study. PLoS One 2018, $13(6)$ :e0197851.

14. Yao J-F, Li N, Jiang J: Clinical Characteristics of Bloodstream Infections in Pediatric Acute Leukemia: A Single-center Experience with 231 Patients. Chin Med J 2017, 130(17):2076-2081.

15. Marin M, Gudiol C, Ardanuy C, Garcia-Vidal C, Calvo M, Arnan M, Carratalà J: Bloodstream infections in neutropenic patients with cancer: differences between patients with haematological malignancies and solid tumours. J Infect 2014, 69(5):417-423.

16. Zhang Y, Lin J, Shi Q, Li C, Liu J, Dai J: Diagnostic accuracy of time to first positivity of blood cultures for predicting severe clinical outcomes in children with pneumonia-related bacteremia. J Investig Med 2020.

17. Chen S, Liu S, Yuan X, Wang H, Wen F: Evaluation of Inflammatory Biomarkers in Pediatric Hematology-Oncology Patients With Bloodstream Infection. J Pediatr Hematol Oncol 2020.

18. Sartori MT, Zurlo C, Bon M, Bertomoro A, Bendo R, Bertozzi I, Radu CM, Campello E, Simioni P, Fabris F: Platelet-Derived Microparticles Bearing PF4 and Anti-GAGS Immunoglobulins in Patients with Sepsis. Diagnostics (Basel) 2020, 10(9).

19. Hannachi N, Lepidi H, Fontanini A, Takakura T, Bou-Khalil J, Gouriet F, Habib G, Raoult D, Camoin-Jau L, Baudoin J-P: A Novel Approach for Detecting Unique Variations among Infectious Bacterial Species in Endocarditic Cardiac Valve Vegetation. Cells 2020, 9(8).

20. Cabanillas Stanchi K, Queudeville M, Malaval C, Feucht J, Schlegel P, Dobratz M, Seitz C, Müller I, Lang P, Handgretinger R et al: Comparison of procalcitonin and C-reactive protein as early diagnostic marker for the identification of transplant-related adverse events after allogeneic hematopoietic stem cell transplantation in pediatric patients. Journal of cancer research and clinical oncology 2019, 145(11):2779-2791.

21. Wang Q, Wang C, Yang M, Li X, Cui J, Wang C: Diagnostic efficacy of serum cytokines and chemokines in patients with candidemia and bacteremia. Cytokine 2020, 130:155081.

22. Fu Y, Chen J, Cai B, Zhang J, Li L, Liu C, Kang Y, Wang L: The use of PCT, CRP, IL-6 and SAA in critically ill patients for an early distinction between candidemia and Gram positive/negative bacteremia. J Infect 2012, 64(4):438-440.

23. Lai L, Lai Y, Wang H, Peng L, Zhou N, Tian Y, Jiang Y, Gong G: Diagnostic Accuracy of Procalcitonin Compared to C-Reactive Protein and Interleukin 6 in Recognizing Gram-Negative Bloodstream Infection: A Meta-Analytic Study. Dis Markers 2020, 2020:4873074.

24. Yu B, Li X, Chen J, Ouyang M, Zhang H, Zhao X, Tang L, Luo Q, Xu M, Yang L et al: Evaluation of variation in D-dimer levels among COVID19 and bacterial pneumonia: a retrospective analysis. J Thromb Thrombolysis 2020.

25. Hu Q, Fu Y, Tang L: Serum D-dimer as a diagnostic index of PJI and retrospective analysis of etiology in patients with PJI. Clinica chimica acta; international journal of clinical chemistry 2020, 506:67-71.

26. Habe K, Wada H, Higashiyama A, Akeda T, Tsuda K, Mori R, Kakeda M, Yamanaka K, Mizutani H: Elevated plasma D-dimer levels in dermatomyositis patients with cutaneous manifestations. Sci Rep 2019, 9(1):1410.

27. Gao Q, Li X, Yu W, Jia F, Yao T, Jin Q, Ji J: Fabrication of Mixed-Charge Polypeptide Coating for Enhanced Hemocompatibility and AntiInfective Effect. ACS Appl Mater Interfaces 2020, 12(2):2999-3010.

\section{Figures}




\section{Distribution of pathogens}
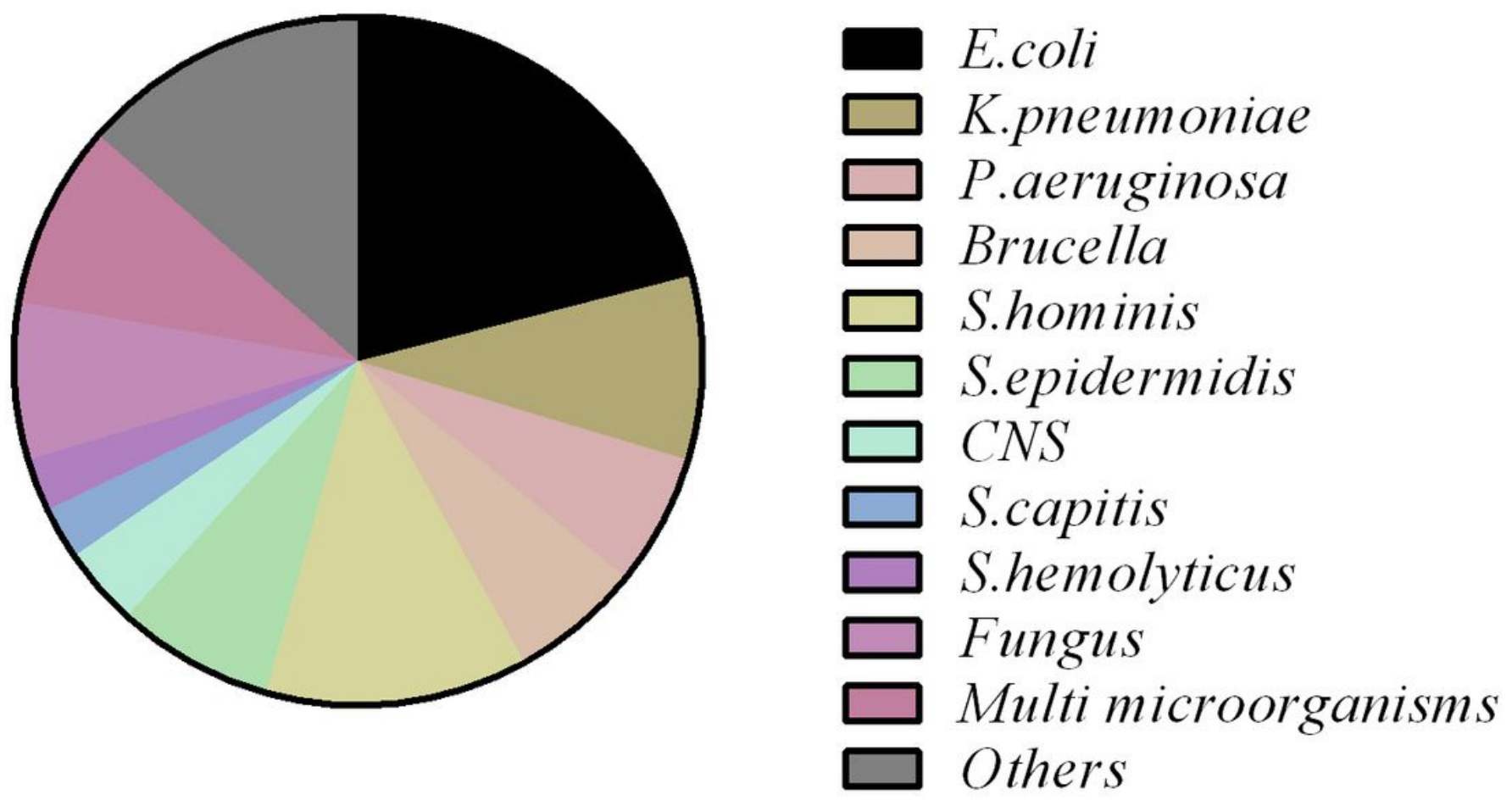

\section{Total $=81$}

Figure 1

Distribution of pathogens in blood culture positive group.
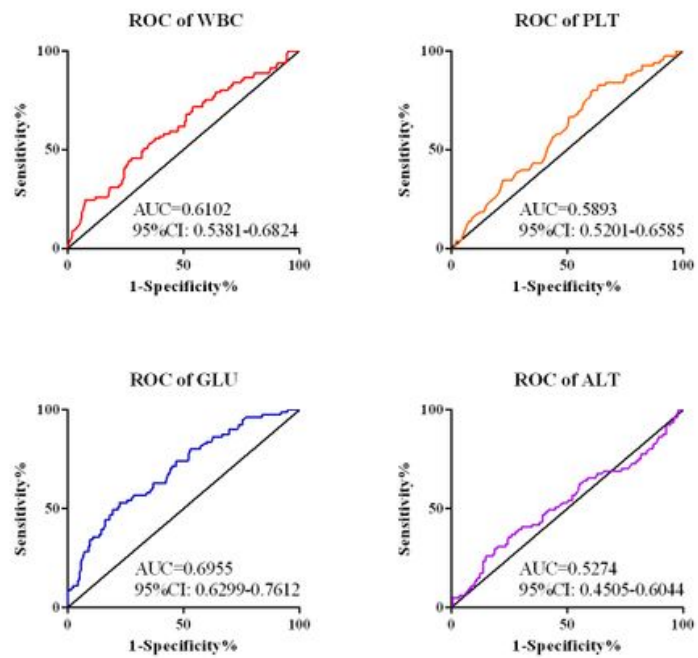

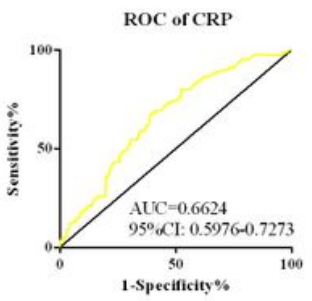

ROC of AST

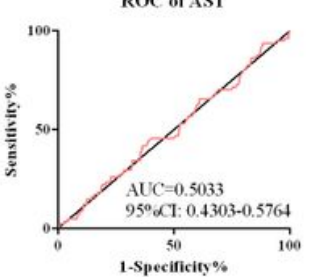

ROC of PCT

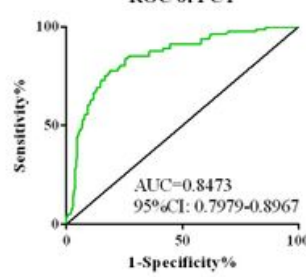

ROC of IL- 6

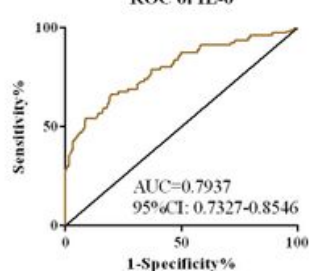

ROC of ALB

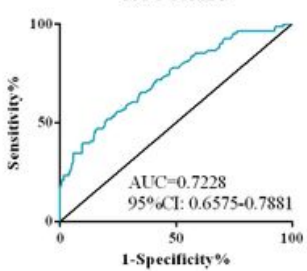

ROC of D-D

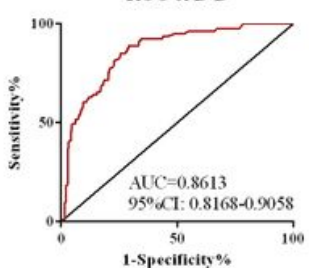

Figure 2

ROC curves of clinical indexes in differentiating infection group and non-infection group. 

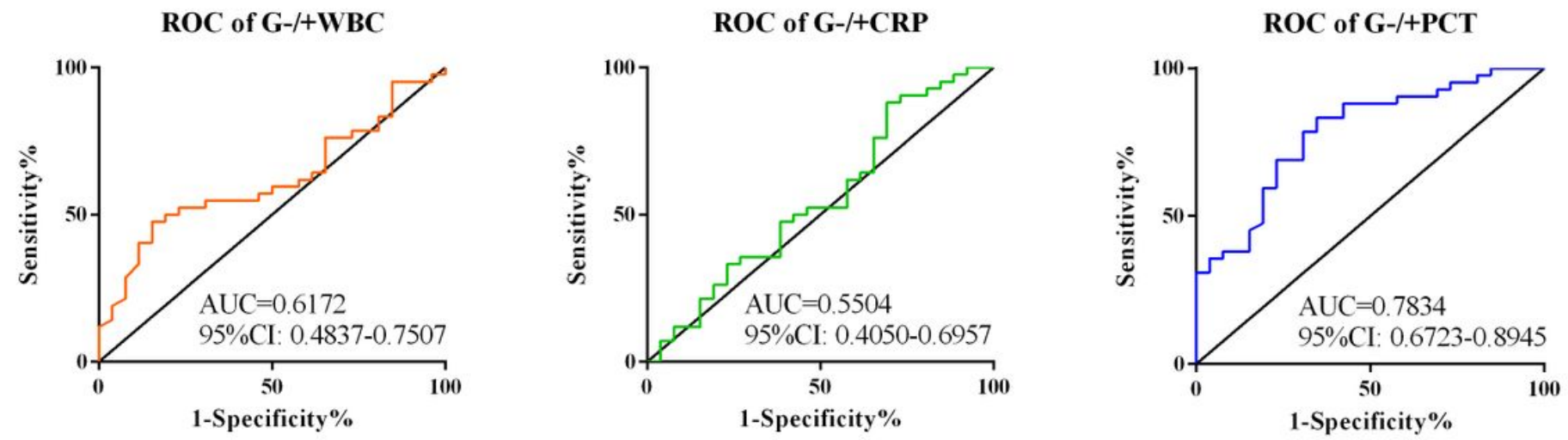

ROC of G-/+IL-6

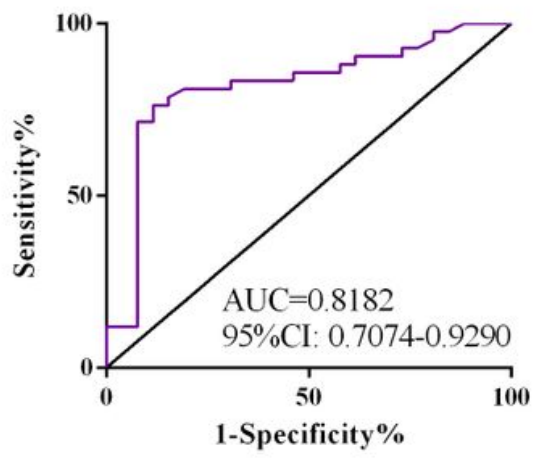

ROC of G-/+ D-D

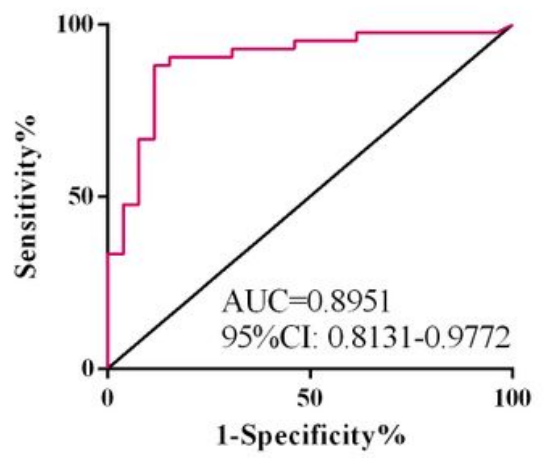

Figure 3

ROC curves of indicators in differentiating Gram- and Gram+ groups. 